Pacific Journal of Mathematics

FUNCTION-THEORETIC DEGENERACY CRITERIA FOR 


\title{
FUNCTION-THEORETIC DEGENERACY CRITERIA FOR RIEMANNIAN MANIFOLDS
}

\author{
Moses GLasner AND Richard KATZ
}

\begin{abstract}
The modulus of a relatively compact set with border consisting of at least two components is a measure of its magnitude with regard to harmonic functions. A divergent modular sum associated with difference sets obtained from an exhaustion of a Riemannian manifold is characteristic of parabolicity. The existence of a divergent minimum modular sum implies that the manifold carries no nonconstant harmonic functions with finite Dirichlet integral.
\end{abstract}

The modular criteria presented in this paper are generalizations of criteria established for Riemann surfaces by Noshiro [6] and Sario [8], [9]. In the two-dimensional case function-theoretic degeneracy is completely determined by the conformal structure, whereas it has been shown by Nakai and Sario [5] that the type of a Riemannian manifold varies when the metric is replaced by a conformally equivalent one. The significance of our result sterns from this fact.

For the completeness of the presentation it is shown that the various characterizations of parabolicity due to Ahlfors, Brelot, Nevanlinna and Ohtsuka remain equivalent in higher dimensions. This overlaps with the work of Itô [2] and Loeb [3] in different settings. A new proof for Riemannian manifolds of the relation, $O_{I I D}=O_{I I B D}$, established in [10] is also given.

1. Let $R$ be an orientable noncompact Riemannian manifold. Let $A \subset R$ and denote by $H(A)$ the class of harmonic functions on $A$ and by $H^{c}(A)$ the functions in $H(A)$ with continuous extensions to $\bar{A}$. For every parametric region $V$ there exists a Green's function $q_{x}^{V}$ with the property $-h(x)=\int_{\partial V} h^{*} d q_{x}^{r}$ for every $h \in H^{c}(V)$. It is well-known that the sheaf of harmonic functions over $R$ satisfies the axioms of a harmonic space and we shall use the standard facts of the theory freely. These together with Green's formulas will serve as our main tools.

2. Consider a fixed parametric region $V \subset R$ and a point $\alpha \in V$. Let $F$ consist of the constant $+\infty$ and of all nonnegative superharmonic functions $s$ on $R$ such that $s \mid V-q_{a}^{V}$ is bounded. Clearly $F$ is a Perron family on $R-\alpha$ and its lower envelope is either $+\infty$ or a function $g_{a}$ harmonic on $R-a$. If the function $g_{a}$ exists it is called the Green's function for $R$. 
Let $\left\{\Omega_{n}\right\}$ be an exhaustion of $R$ by regular regions. Let $t_{n} \in H^{c}\left(\Omega_{n}-\bar{\Omega}_{0}\right)$ with $t_{n}\left|\partial \Omega_{0}=0, t_{n}\right| \partial \Omega_{n}=1$. The functions $t_{n}$ form a decreasing sequence and the harmonic function $t=\lim _{n} t_{n}$ on $R-\bar{\Omega}_{0}$ is called the harmonic measure of the ideal boundary of $R$ with respect to $\Omega_{0}$.

3. The above definitions are related as follows.

THEOREM. Conditions (a), (b) and (c) are equivalent.

(a) There exists a nonconstant positive superharmonic function on $R$.

(b) $t$ is not identically zero.

(c) $R$ does not belong to the class $O_{G}$ of manifolds for which $g_{a}$ does not exist.

It is obvious that either (b) or (c) implies (a). To show that (a) implies (b), suppose that $t \equiv 0$ and let $s$ be a positive superharmonic function. Set $m=\min _{\partial \Omega_{0}} s=\min _{\bar{\Omega}_{0}} s$ and observe that $s \geqq m\left(1-t_{n}\right)$ on $\bar{\Omega}_{n}-\bar{\Omega}_{0}$. On letting $n$ tend to $\infty$ we conclude that $s \geqq m$ on $R$ and hence it is a constant. Now suppose that (a) is true. Then (b) holds for all choices of $\Omega_{0}$ and in particular for the choice

$$
\Omega_{0}=\left\{x \mid q_{a}^{V}(x)>1\right\} \subset V .
$$

Set $\alpha=\left(\min _{\partial V} t\right)^{-1}$. By virtue of the fact that $q_{a}^{V} \mid \partial V=0$ we conclude that the function

$$
q(x)=\left\{\begin{array}{l}
q_{a}^{V}(x), \quad x \in \Omega_{0} \\
1-2 \alpha t(x), \quad x \in R-\Omega_{0}
\end{array}\right.
$$

is superharmonic on $R$ and bounded from below by $1-2 \alpha$. Thus $F$ contains more than one element and consequently $R \notin O_{G}$.

The modulus $\tau_{n}$ of the region $\Omega_{n}-\bar{\Omega}_{0}$ is by definition $\left(\int_{\partial \Omega_{0}} * d t_{n}\right)^{-1}$ and we can state the following immediate

Corollary. The constants $\tau_{n}$ tend to $+\infty$ if and only if $R \in O_{G}$.

4. In addition to the above notions we define the modulus function $\omega_{i} \in H^{c}\left(\Omega_{i}-\bar{\Omega}_{i-1}\right)$ by the conditions $\omega_{i}\left|\partial \Omega_{i-1}=0, \omega_{i}\right| \partial \Omega_{i}=\mu_{i}$ (const.) and $\int_{\partial \Omega_{i-1}} * d \omega_{i}=1$. The constant $\mu_{i}$ is called the modulus of $\Omega_{i}-\bar{\Omega}_{i-1}$. We now generalize the $O_{G}$ modular criterion of Noshiro [6] and Sario [9] to Riemannian manifolds.

THeOREM. There exists an exhaustion $\left\{\Omega_{n}\right\}$ of $R$ such that $\sum \mu_{n}=+\infty$ if and only if $R \in O_{G}$. 
Suppose that such an exhaustion exists. The Dirichlet integral of $w_{n}=\tau_{n} t_{n}$ over $\Omega_{n}-\bar{\Omega}_{0}$ is

$$
D_{n}\left(w_{n}\right)=\int_{\Omega_{n}-\overline{\Omega_{0}}} d w_{n} \wedge * d w_{n}=\int_{\partial \Omega_{n}-\partial \Omega_{0}} w_{n}^{*} d w_{n}=\tau_{n} .
$$

On the other hand, $D_{n}\left(w_{n}\right)$ is equal to the sum $\sum_{n=1}^{n} D_{i}\left(w_{n}\right)$ of Dirichlet integrals over $\Omega_{i}-\bar{\Omega}_{i-1}$. Using the Schwarz inequality we obtain

$$
D_{i}\left(w_{n}\right) \geqq \frac{D_{i}^{2}\left(\omega_{i}, w_{n}\right)}{D_{\imath}\left(\omega_{i}\right)}=\frac{\left(\int_{\partial \Omega_{i}-\partial \Omega_{i-1}} \omega_{i}^{*} d w_{n}\right)^{2}}{\int_{\partial \Omega_{i}-\partial \Omega_{i-1}} \omega_{i}^{*} d \omega_{i}}=\frac{\mu_{i}^{2}}{\mu_{i}} .
$$

Thus $\tau_{n} \geqq \sum_{i=1}^{n} \mu_{2}$, and consequently $\tau_{n} \rightarrow+\infty$. This implies, by Corollary 3 , that $R \in O_{G}$.

Conversely, suppose that $R \in O_{G}$ and $\left\{\Omega_{n}\right\}$ is any exhaustion of $R$. Then there exists an $n_{1}$ such that $\tau_{n_{1}}>1$. Then $\left\{\tau_{n}^{\prime}\right\}$ constructed for the exhaustion $\left\{\Omega_{n}\right\}_{n_{1}}^{\infty}$ also tend to $+\infty$ and a fortiori there is an $n_{2}$ with $\tau_{n_{2}}^{\prime}>1$. Proceeding in this fashion we obtain an exhaustion $\left\{\Omega_{n_{i}}\right\}$ such that the modular sum

$$
\sum_{i=1}^{\infty} \mu_{i}=\sum_{i=1}^{\infty} \tau_{n_{i}}^{(i-1)}=+\infty .
$$

The above condition is not necessary in the sense that every manifold has an exhaustion with arbitrarily modular sum (cf. [1]).

5. We now turn to a criterion for membership in the class $O_{H D}$ of manifolds on which every member of the class $H D(R)$ of harmonic functions with finite Dirichlet integral over $R$ is constant. The Dirichlet integral of an $f \in C^{1}(R)$ over $R$ is, by definition,

$$
D(f)=\lim _{n \rightarrow \infty} D_{n}(f),
$$

where $D_{n}(f)$ is taken over $\Omega_{n}$ and $\left\{Q_{n}\right\}$ is an exhaution of $R$. Clearly $D(f)$ is independent of the choice of the exhaustion $\left\{\Omega_{n}\right\}$ and

$$
D(f)<+\infty, D(g)<+\infty
$$

imply the existence of the limit $D(f, g)=\lim _{n \rightarrow \infty} D_{n}(f, g)$.

First we prove the

THEOREM. There exists a nonconstant element of $H D(R)$ if and only if there exists one which is also bounded. That is, $O_{I I D}=O_{I I B D}$.

Let $u$ be a nonconstant element of $H D(R)$ and set

$$
u_{m}=\min (m, \max (u,-m)) \text {. }
$$


Denote by $v_{m n}$ the continuous function on $R$ such that $v_{m n}=u_{m}$ on $R-\Omega_{n}$ and $v_{m n}$ is harmonic on $\Omega_{n}$. Since $\left\{v_{m n}\right\}_{n=0}^{\infty}$ is uniformly bounded it has a subsequence also denoted by $\left\{v_{m n}\right\}_{n=0}^{\infty}$ which converges to a harmonic function $v_{m}$ uniformly on compact subsets. By Green's formula we obtain $D\left(u_{m}-v_{m n}\right)=D\left(u_{m}\right)-D\left(v_{m n}\right) \geqq 0$ and

$$
D\left(v_{m n}-v_{m, n+p}\right)=D\left(v_{m n}\right)-D\left(v_{m, n+p}\right) \geqq 0 .
$$

From this we see that $d=\lim _{n} D\left(v_{m n}\right)$ exists. By Fatou's lemma we also have $D\left(v_{m n}-v_{m}\right) \leqq D\left(v_{m n}\right)-d$ and consequently $\lim _{n} D\left(v_{m n}-v_{m}\right)=0$.

For every $m, v_{m} \in H B D(R)$ and we shall complete proof by showing that the assumption $v_{m}$ is a constant for every $m$ leads to a contradiction. Set $g_{m n}=u_{m}-v_{m n}, g_{n}=\lim _{n} g_{m n}=u_{m}-v_{m}$ and note that $\lim _{n} D\left(g_{m n}-u_{m}\right)=0$. Since $\operatorname{supp} g_{m n} \subset \bar{\Omega}_{n}$, we have

$$
D\left(g_{m n}, u\right)=\int_{\partial \Omega_{n}} g_{m n} * d u=0 \text {. }
$$

Thus $D\left(u_{m}, u\right)=\lim _{n} D\left(g_{m n}, u\right)=0$ and $D(u)=\lim _{m} D\left(u_{m}, u\right)=0$, which contradicts the choice of $u$. Our proof stems in spirit from Royden [7].

6. Sario [8] introduced a sufficient condition for a Riemann surface not to carry nonconstant analytic functions with finite Dirichlet integral. Since analytic, as such, has no meaning in Riemannian manifolds we replace Sario's result with an $O_{H D}$ criterion.

In general the open sets $\Omega_{n}-\bar{\Omega}_{n-1}$ will consist of components $E_{i n}$, $i=1, \cdots, i(n)$. Let $\omega_{i n}$ denote the modulus function of $E_{i n}$, i.e., $\omega_{i n} \in H^{c}\left(E_{i n}\right), \omega_{i n}\left|\partial E_{i n} \cap \partial \Omega_{n-1}=0, \omega_{i n}\right| \partial E_{i n} \cap \partial \Omega_{n}=\mu_{i n} \quad$ (const.) such that $\int * d \omega_{i n}=1$. Set $\nu_{n}=\min _{i} \mu_{i n}$.

THEOREM. If $\left\{\Omega_{n}\right\}$ is an exhaustion of $R$ with a divergent minimum modular sum $\sum \nu_{n}=+\infty$, then $R \in O_{H D}$.

Since Euclidean $n$-space $(n \geqq 3)$ is not in $O_{G}$ but in $O_{H D}$, Theorem 4 shows that the divergence of the minimum modular sum is not a necessary condition for a manifold to be in $O_{H D}$.

For the proof of the theorem let $u$ be a nonconstant harmonic function on $R$ with $D(u)<+\infty$; by Theorem 5 we may assume that $|u| \leqq M$. We further normalize $u$ by adding a constant so that

$$
\int_{\partial \Omega_{0}} u * d u=0 \text {. }
$$

By a theorem of Morse (cf. Milnor [4]) $\omega_{n}$ and its gradient can be uniformly approximated by $C^{\infty}$ functions $h$ and their gradients, where the $h$ 's have the same boundary values as $\omega_{n}$ and grad $h=0$ only at 
a finite number of points. We choose such an $h$ with the property that

$$
\int_{\beta_{n i}}|\operatorname{grad} h| d S \leqq 2,
$$

where $\beta_{n \lambda}$ is the level surface $h=\lambda, 0<\lambda<\mu_{n}$.

Denote by $D_{n \lambda}(u)$ the Dirichlet integral of $u$ over the open set bounded by $\partial \Omega_{0}$ and $\beta_{n \lambda}$. By the definition of gradient and the Schwarz inequality we obtain

$$
\frac{d}{d \lambda} D_{n \lambda}(u)=\int_{\beta_{n \lambda} \lambda} \frac{|\operatorname{grad} u|^{2}}{|\operatorname{grad} h|} d s \geqq \frac{\left(\int_{\beta_{n \lambda}}|\operatorname{grad} u| d S\right)^{2}}{\int_{\beta_{n \lambda}}|\operatorname{grad} h| d S} \geqq \frac{1}{2}\left(\int_{\beta_{n \lambda}}|* d u|\right)^{2},
$$

except for a finite number of values of $\lambda$. On the other hand, Green's formula gives

$$
D_{n \lambda}(u)=\int_{\beta_{n} \lambda} u * d u \leqq M \int_{\beta_{n \lambda}}|* d u| \cdot
$$

We conclude that

$$
\frac{d}{d \lambda} D_{n \lambda}(u) \geqq \frac{1}{2 M^{2}} D_{n \lambda}^{2}(u) .
$$

We now integrate this inequality for $n \geqq 2$ :

$$
\int_{0}^{\nu_{n}} \frac{\frac{d}{d \lambda} D_{n \lambda}(u)}{D_{n \lambda}(u)} d \lambda \geqq \frac{1}{2 M^{2}} \int_{0}^{\nu_{n}} D_{n \lambda}(u) d \lambda \geqq \nu_{n} a,
$$

where $a=\left(2 M^{2}\right)^{-1} D_{1}(u)$. This implies that

$$
D_{n}(u) \geqq D_{n, \nu_{n}}(u) \geqq D_{n-1}(u) \exp \left(\nu_{n} a\right)
$$

and iteration gives $D_{n}(u) \geqq D_{1}(u) \exp \left(a \sum_{2}^{a} \nu_{n}\right)$. Since $D(u)<+\infty$, we conclude that $\sum_{1}^{\infty} \nu_{n}<+\infty$.

\section{REFERENCES}

1. M. Glasner, R. Katz and M. Nakai, Bisection into small annuli, Pacific J. Math. 24 (1968), 457-461.

2. S. Itô, On existence of Green function and positive superharmonic functions for linear elliptic operators of second order, J. Math. Soc. Japan 16 (1964), 299-306.

3. P. A. Loeb, An axiomatic treatment of pairs of elliptic differential operators, Ann. Inst. Fourier Grenoble 16 (1966), 167-208.

4. J. Milnor, Lectures on the h-cobordism theorem, Princeton Univ. Press, Princeton, N. J., 1965.

5. M. Nakai and L. Sario, Classification and deformation of Riemannian spaces, Math. Scand. 20 (1967), 193-208. 
6. K. Noshiro, Open Riemann surface with null boundary, Nagoya Math. J. 3 (1951), 73-79.

7. H. L. Royden, Harmonic functions on open Riemann surfaces, Trans. Amer. Math. Soc. 73 (1952), 40-94.

8. L. Sario, Riemannsche Flächen mit hebbarem Rand, Ann. Acad. Sci. Fenn. Ser A. I. 50 (1948).

9. —- Modular criteria on Riemann surfaces, Duke Math. J. 20 (1953), 279-286. 10. L. Sario, M. Schiffer and M. Glasner, The span and principal functions in Riemannian spaces, J. Analyse Math. 15 (1965), 115-134.

Received February 13, 1968.

California Institute of Technology

Pasadena, California

California State College

Los Angeles, California 


\section{PACIFIC JOURNAL OF MATHEMATICS}

\section{EDITORS}

H. ROYDEN

Stanford University

Stanford, California

\section{R. $R$ PHELPS}

University of Washington

Seattle, Washington 98105

\section{J. DugunduI}

Department of Mathematics

University of Southern California

Los Angeles, California 90007

RICHARD ARENS

University of California

Los Angeles, California 90024

\section{ASSOCIATE EDITORS}

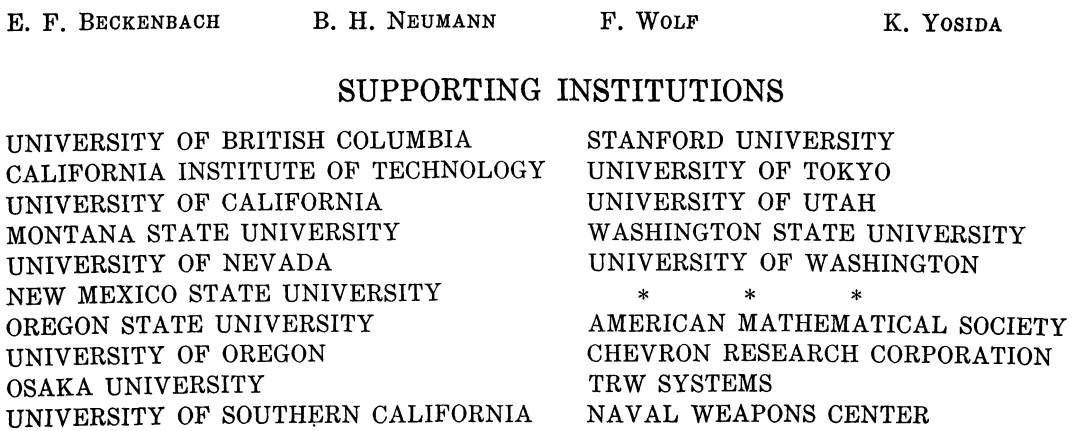

The Supporting Institutions listed above contribute to the cost of publication of this Journal, but they are not owners or publishers and have no responsibility for its content or policies.

Mathematical papers intended for publication in the Pacific Journal of Mathematics should be in typed form or offset-reproduced, double spaced with large margins. Underline Greek letters in red, German in green, and script in blue. The first paragraph or two must be capable of being used separately as a synopsis of the entire paper. It should not contain references to the bibliography. Manuscripts, in duplicate if possible, may be sent to any one of the four editors. Please classify according to the scheme of Math. Rev. 36, 1539-1546. All other communications to the editors should be addressed to the managing editor, Richard Arens, University of California, Los Angeles, California, 90024.

50 reprints are provided free for each article; additional copies may be obtained at cost in multiples of 50 .

The Pacific Journal of Mathematics is published monthly. Effective with Volume 16 the price per volume (3 numbers) is $\$ 8.00$; single issues, $\$ 3.00$. Special price for current issues to individual faculty members of supporting institutions and to individual members of the American Mathematical Society: $\$ 4.00$ per volume; single issues $\$ 1.50$. Back numbers are available.

Subscriptions, orders for back numbers, and changes of address should be sent to Pacific Journal of Mathematics, 103 Highland Boulevard, Berkeley, California, 94708.

PUBLISHED BY PACIFIC JOURNAL OF MATHEMATICS, A NON-PROFIT CORPORATION

Printed at Kokusai Bunken Insatsusha (International Academic Printing Co., Ltd.), 7-17, Fujimi 2-chome, Chiyoda-ku, Tokyo, Japan. 


\section{Pacific Journal of Mathematics}

Vol. 28, No. $2 \quad$ April, 1969

Richard Arens and Donald George Babbitt, The geometry of relativistic

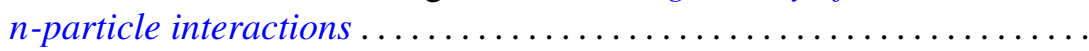

Kirby Alan Baker, Hypotopological spaces and their embeddings in lattices

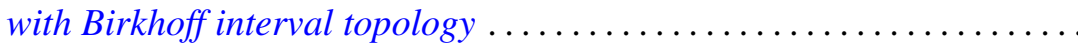

J. Lennart (John) Berggren, Finite groups in which every element is conjugate to its inverse ........................... 289

Beverly L. Brechner, Homeomorphism groups of dendrons . . . . . . . . . . . 295

Robert Ray Colby and Edgar Andrews Rutter, QF - 3 rings with zero singular ideal ................................. 303

Stephen Daniel Comer, Classes without the amalgamation property....... 309

Stephen D. Fisher, Bounded approximation by rational functions ......... 319

Robert Gaines, Continuous dependence for two-point boundary value problems..................................... 327

Bernard Russel Gelbaum, Banach algebra bundles ............... 337

Moses Glasner and Richard Emanuel Katz, Function-theoretic degeneracy criteria for Riemannian manifolds ...................... 351

Fletcher Gross, Fixed-point-free operator groups of order $8 \ldots \ldots \ldots \ldots 357$

Sav Roman Harasymiv, On approximation by dilations of distributions . . . . 363

Cheong Seng Hoo, Nilpotency class of a map and Stasheff's criterion ... . . 375

Richard Emanuel Katz, A note on extremal length and modutus.......... 381

H. L. Krall and I. M. Sheffer, Difference equations for some orthogonal polynomials ................................

Yu-Lee Lee, On the construction of lower radical properties ........... 393

Robert Phillips, Liouville's theorem........................... 397

Yum-Tong Siu, Analytic sheaf cohomology groups of dimension $n$ of

n-dimensional noncompact complex manifolds ..... . .

Michael Samuel Skaff, Vector valued Orlicz spaces. II...

James DeWitt Stein, Homomorphisms of $B^{*}$-algebras .... . .

Mark Lawrence Teply, Torsionfree injective modules .... . . .

Richard R. Tucker, The $\delta^{2}$-process and related topics. II .

David William Walkup and Roger Jean-Baptiste Robert Wets, Lifting

projections of convex polyhedra...

Thomas Paul Whaley, Large sublattices of a lattice. 